\title{
EUROPA AL BIVIO \\ Recensione a La crisi dell'integrazione europea e la Turchia. Una analisi istituzionalista ed una critica federalista*
}

Questo non voluminoso, ma denso libro di Maurizio Mistri, docente di Economia Internazionale alla Facoltà di Scienze Politiche dell'Università di Padova, affronta la spinosa e controversa questione dell'eventuale adesione della Turchia all'Unione europea, considerata come una questione emblematica del continuo processo di allargamento vissuto nei decenni passati dalla stessa Unione per cui adesso essa è giunta a comprendere come noto - 27 paesi.

Infatti, l'autore inquadra la vicenda del rapporto fra Turchia ed Ue all'interno del progressivo mutamento di approccio al modo in cui è finora avvenuto il processo d'integrazione europea e dal quale, a suo avviso, ne è uscita sconfitta la visione federalista, che era orientata all'istituzione degli Stati Uniti d'Europa. Da tale mutamento di approccio ne sembra uscita vincitrice invece la visione "funzionalista", la quale è piuttosto orientata, prescindendo dai nobili ideali e dai lungimiranti principi dei padri fondatori della Comunità europea, alla creazione di una più semplice quanto più concreta entità istituzionale, denominata "mercati uniti d'Europa" e volta soprattutto al conseguimento di vantaggi commerciali, economici e finanziari da parte dei singoli Stati membri.

La tesi sostenuta dall'autore è che, se si intende perseguire un vero processo integrativo fra i paesi che attualmente fanno parte dell'Ue, occorre che a farsene carico siano soprattutto quei paesi che - oltre a condividere una comune cultura politica - sono anche tra loro omogenei in termini di strutture economiche e per visione della governance in materia di politica economica, così come nel caso dell'Unione monetaria europea. In seguito ai continui allargamenti dell'Unione europea, sostenuti soprattutto da paesi come il Regno Unito, l'originario afflato europeistico è andato spegnendosi, anche perché molti dei paesi nuovi entrati - come quelli cui spetterà la presidenza di turno nel corso di quest'anno - sono paradossalmente "euroscettici"; alcuni di essi poi si sono persino dimostrati più che altro interessati a ottenere - attraverso la funzione redistributrice e riequilibratrice

* Mistri M. (2010), La crisi dell'integrazione europea e la Turchia. Una analisi istituzionalista ed una critica federalista, Edizioni La Gru, Padova, pagine 156. 
svolta dal bilancio comunitario - risorse finanziarie provenienti dai paesi europei economicamente più avanzati.

Allora si pongono alcune domande cui Mistri cerca di fornire adeguate risposte nel suo libro.

Una prima domanda è se i cittadini europei e i loro governanti sono ancora seriamente interessati a conseguire l'integrazione politica del loro continente o se, invece, si accontentano di una forma d'integrazione economica, più o meno stretta. Se prevale quest'ultimo orientamento, i cittadini europei devono rendersi conto, però, che un'integrazione economica, priva di un'integrazione politica, nel lungo periodo è destinata al collasso istituzionale. Se invece gli europei sono ancora a favore dell'integrazione politica del Vecchio Continente, essi devono anche chiedersi se i continui allargamenti che si sono registrati finora e quelli che sono previsti per il prossimo futuro a favore soprattutto dei paesi dei Balcani, siano effettivamente funzionali a tale obiettivo o se, invece, finiscano solo per vanificarlo.

La questione dell'ingresso della Turchia nell'Europa viene attentamente esaminata in tale contesto logico, partendo dal punto di vista europeo.

Ovviamente, però, si considera anche il modo in cui la Turchia cerca di vivere il proprio processo di modernizzazione in funzione di tale ingresso. Il partito islamico dell' attuale Presidente Erdogan, che può essere considerato un leader di grandi capacità politiche, sta imprimendo una svolta che va via via prescindendo dal ruolo svolto dall'Ue nel contesto internazionale e che tende invece a valorizzare il patrimonio della tradizione culturale della Turchia, sebbene su di esso si cerchi d'innestare un più originale processo di sviluppo. D'altro canto, l'eventualità di un abbandono dell'opzione europea, che non va esclusa a priori, per la Turchia non dovrebbe essere intesa come una drammatica sconfitta politica, bensì come l'espressione di una più coraggiosa ricerca di un'autonoma via allo sviluppo sociale ed economico, che magari potrebbe essere addirittura presa ad esempio dagli altri paesi che si affacciano sulla sponda meridionale del Mediterraneo.

Aurelio Bruzzo 